\title{
Farmacokinetiek van geneesmiddelen bij vogels en de toepassingen en beperkingen van dosisextrapolatie
}

\author{
Pharmacokinetics of drugs in avian species and the applications \\ and limitations of dose extrapolation
}

\author{
${ }^{1}$ R. Houben, ${ }^{1,2}$ G. Antonissen, ${ }^{1}$ S. Croubels, ${ }^{1} \mathrm{P}$. De Backer, ${ }^{1} \mathrm{M}$. Devreese \\ ${ }^{1}$ Vakgroep Farmacologie, Toxicologie en Biochemie, \\ ${ }^{2}$ Vakgroep Pathologie, Bacteriologie en Pluimveeziekten \\ Faculteit Diergeneeskunde, Universiteit Gent, \\ Salisburylaan 133, B-9820 Merelbeke \\ Gunther.Antonissen@UGent.be \\ Mathias.Devreese@UGent.be
}

\section{AMENVATTING}

De farmacokinetische processen van geneesmiddelen, i.e. absorptie, distributie, metabolisatie en excretie, kunnen sterk verschillen tussen vogels en zoogdieren. Zo worden aminoglycosiden bij vogels trager geklaard en hebben een lager distributievolume dan bij zoogdieren. Deze farmacokinetische verschillen zijn hoofdzakelijk het gevolg van anatomische, biochemische en fysiologische verschillen tussen vogels en zoogdieren. Deze diersoortverschillen en verschillen in geobserveerde en voorspelde farmacokinetische parameters van geneesmiddelen bij vogels geëxtrapoleerd vanuit zoogdieren, zoals de klaring voor enrofloxacine, salicylzuur, meloxicam en flunixine, wijzen erop dat farmacokinetische gegevens van een geneesmiddel bij zoogdieren nauwelijks geëxtrapoleerd kunnen worden naar vogels. Ook tussen vogelsoorten onderling kunnen er verschillen aanwezig zijn. Indien mogelijk dient de dosisselectie van een geneesmiddel voor vogels bijgevolg te gebeuren op basis van de farmacokinetische gegevens van de desbetreffende vogelsoort. Indien dergelijke gegevens niet beschikbaar zijn, kan er gebruik gemaakt worden van allometrische schaling, waarbij de dosisselectie gebeurt op basis van de farmacokinetische gegevens van een andere vogelsoort gecorreleerd aan het lichaamsgewicht van deze vogelsoorten. Schaling op basis van zoogdiergegevens wordt slechts aangeraden indien de farmacokinetische gegevens van andere vogelsoorten niet beschikbaar zijn en het betreffende geneesmiddel een lage toxiciteit heeft.

\section{ABSTRACT}

The pharmacokinetic processes of drugs, i.e. absorption, distribution, metabolization and elimination, differ between birds and mammals. For instance, the clearance and volume of the distribution of aminoglycosides are lower in birds than in mammals. These pharmacokinetic differences are caused by differences in anatomy, biochemistry and physiology between birds and mammals. The species differences in pharmacokinetics and differences in the observed and predicted pharmacokinetic parameters of some drugs in birds after extrapolation from mammals (e.g. clearance of enrofloxacin, salicylic acid, meloxicam and flunixin), show that pharmacokinetic data on mammals can hardly be extrapolated to birds as such. Furthermore, differences in pharmacokinetic behavior between bird species exist as well. Therefore, the selection of an appropriate posology for birds has to be based on pharmacokinetic data of that specific bird species. In absence of these data, dose selection might be done using allometric scaling. The pharmacokinetic data are scaled in correlation to the body weight of a different bird species. In case these data are also not available, dose selection using allometric scaling with pharmacokinetic data from mammals should preferably only be done for drugs with a low toxicity. 


\section{INLEIDING}

Het behandelen van zowel zoogdieren, vogels, vissen, reptielen als amfibieën is voor dierenartsen vaak een uitdaging. Verschillen in anatomie, biochemie en fysiologie tussen deze diersoorten maken het opstellen van een correct doseringsschema voor geneesmiddelen vaak complex. Verschillen tussen klassen van dieren, zoals Aves, Mammalia, alsook binnen eenzelfde klasse kunnen de farmacokinetiek en farmacodynamiek van de geneesmiddelen beïnvloeden (Dorrestein, 1991; Toutain et al., 2010).

De beschikbaarheid van geregistreerde geneesmiddelen voor het gebruik bij vogels is bovendien beperkt, waardoor dierenartsen genoodzaakt zijn om geneesmiddelen te gebruiken die geregistreerd zijn voor een andere aandoening bij dezelfde diersoort of voor een andere diersoort ("off label use") (Hunter et al., 2008). Allometrische schaling is een extrapolatiemethode voor onder andere farmacokinetische parameters, waarbij gebruik gemaakt wordt van het lichaamsgewicht van het dier, zodat dosisselectie mogelijk is zonder dat men moet beschikken over diersoortspecifieke farmacokinetische gegevens (Boxenbaum en Fertig, 1984; Lave et al., 1997; Obach et al., 1997). Ondanks deze schalingsmogelijkheden dient finaal elk geneesmiddel bij elke diersoort onderzocht te worden om een effectief en veilig gebruik te garanderen (Toutain et al., 2010). In dit artikel wordt een comparatief overzicht gegeven van de verschillen in farmacokinetiek van een aantal geneesmiddelen bij verschillende vogelsoorten om op deze wijze de praktijkdierenarts te helpen een correcte posologie voor bepaalde geneesmiddelen bij verschillende vogelsoorten te hanteren.

\section{BEGINSELEN VAN DE FARMACOKINETISCHE PROCESSEN}

Speciesafhankelijke verschillen in farmacokinetiek kunnen het gevolg zijn van verschillen in absorptie, distributie en/of eliminatie, bestaande uit biotransformatie en excretie (Baert, 2003; Jerzsele, 2012).

Oraal toegediende geneesmiddelen ondergaan verschillende processen voordat ze terechtkomen in de systemische circulatie, namelijk vrijstelling uit de doseringsvorm, passage naar het darmlumen, transport doorheen de intestinale mucosa en via de vena-portapassage doorheen de lever. Er kan reeds eliminatie van het geneesmiddel optreden bij passage door deze weefsels, ook wel het "first-pass"-effect genoemd (Rowland en Tozer, 1995). Dit effect is afwezig bij parenterale toediening (Shargel et al., 2004). Het "first-pass"-effect kan de biologische beschikbaarheid van een geneesmiddel sterk verlagen. De biologische beschikbaarheid is de mate en snelheid waarin een geneesmiddel, toegediend onder een bepaalde vorm, onveranderd de systemische circulatie bereikt (Gibaldi en Perrier, 1975; Rowland en Tozer, 1995).
Distributie is het proces van de verdeling van een geneesmiddel van de systemische circulatie naar verschillende weefsels, inclusief doelwit- en eliminatieorganen (Rowland en Tozer, 1995). De mate van distributie van een geneesmiddel wordt weergegeven door het schijnbaar distributievolume (Vd) en is afhankelijk van de distributiesnelheid en de mate van eiwitbinding. Het distributievolume is de constante verhouding tussen de totale hoeveelheid geneesmiddel in het lichaam (X) en de plasmaconcentratie van een geneesmiddel $(\mathrm{Cp})$ op datzelfde tijdstip $(\mathrm{Vd}=\mathrm{X} /$ $\mathrm{Cp}$ ), nadat het distributie-evenwicht is bereikt ( $\mathrm{Ri}$ viere, 2011).

Eliminatie is het verwijderen van een geneesmiddel uit het lichaam via biotransformatie en/of excretie. Via biotransformatie, ook wel metabolisatie genoemd, wordt het geneesmiddel chemisch omgevormd tot een metaboliet. Dit gebeurt voornamelijk in de lever, maar is ook mogelijk in de nieren, huid, longen en maagdarmmucosa. Door excretie wordt het geneesmiddel en/of de metabolieten uitgescheiden via de nier en/of gal en in mindere mate via de speekselklieren, longen en huid (Shargel et al., 2004).

De eliminatiehalfwaardetijd $\left(\mathrm{T}_{1 / 2 \mathrm{el}}\right)$ is de tijd die het lichaam nodig heeft om de helft van het geneesmiddel te elimineren. Deze wordt bepaald door het distributievolume en de klaring $(\mathrm{Cl})$ en wordt weergegeven als de volgende formule: $T_{1 / 2 \mathrm{el}}=\frac{0.693 * \mathrm{Vd}}{\mathrm{Cl}}$

De efficiëntie van eliminatie van het geneesmiddel wordt weergegeven door de klaring die wordt gedefinieerd als het plasmavolume geklaard van het geneesmiddel door eliminatieprocessen per tijdseenheid.De totale klaring omvat de som van de klaring van het geneesmiddel door alle eliminatieorganen, zoals lever, nieren en longen (Shargel et al., 2004).

ENKELE SPECIFIEKE ANATOMISCHE ASPECTEN VAN VOGELS DIE EEN INVLOED KUNNEN HEBBEN OP DE FARMACOKINETIEK BIJ VOGELS

De ledigingssnelheid van de krop is afhankelijk van de vullingsgraad en consistentie van de inhoud en kan het absorptiepatroon van oraal toegediende geneesmiddelen beïnvloeden. Daarnaast kunnen de krop $\mathrm{pH}$ en de aanwezigheid van Lactobacillus spp. als onderdeel van de microbiota van de krop aanleiding geven tot precipitatie en/of inactivatie van het geneesmiddel en zo de absorptie verlagen (Devriese en Dutta, 1984). Andere anatomische verschillen zijn aanwezig ter hoogte van de maag, die bij vogels bestaat uit een kliermaag en spiermaag, en het duodenum, dat bij vogels korter is en tevens een microbiota bevat die bepaalde geneesmiddelen kan inactiveren. Algemeen kan worden aangenomen dat geneesmiddelen die worden toegediend in tabletvorm, capsules en andere vaste toedieningsvormen in de spiermaag snel 
worden gedesintegreerd, waarna een snelle passage volgt van de actieve component naar de dunne darm. Geneesmiddelen toegediend in oplossing passeren snel het proximale deel van het spijsverteringsstelsel om snel na toediening terecht te komen in de dunne darm (Dorrestein en Van Miert, 1988; Vermeulen et al., 2002).

De renale excretie bij vogels verschilt van deze bij zoogdieren wegens de aanwezigheid van zowel reptieltype- als zoogdiertype-nefronen in de glomerulus. Reptieltype-nefronen bevatten glomerulaire capillairen met een kleiner filtratie-oppervlak, wat de glomerulaire filtratiesnelheid kan beïnvloeden. Bovendien ontbreekt de lis van Henle, zodat het concentrerend vermogen van de nier bij vogels beperkt is daar slechts $20-30 \%$ van de nefronen van het zoogdiertype is (Braun en Dantzler, 1972). Tubulaire reabsorptie van geneesmiddelen is bij vogels beperkt tot afwezig (Vermeulen et al., 2002). Het renaal-portaalsysteem kan er ook voor zorgen dat geneesmiddelen intramusculair toegediend in de achterste lichaamshelft, voornamelijk in de bilspier, direct door de nieren worden uitgescheiden zonder terecht te komen in de systemische circulatie. Of en in welke mate dit gebeurt, is afhankelijk van de hydratatietoestand van het dier (Akester, 1967; Goldstein en Skadhauge, 2000; Vermeulen et al., 2002).

\section{SPECIESVERSCHILLEN IN DISTRIBUTIE EN ELIMINATIE}

Kennis van de farmacokinetiek is belangrijk voor de posologie van geneesmiddelen (Lees en AliAbadi, 2002). Echter, farmacokinetische gegevens voor gezelschapsvogels zijn beperkt, met uitzondering van onder andere enkele niet-steroïdale ontstekingsremmers (NSAIDs), aminoglycosiden en carboplatine.

Het distributievolume van aminoglycosiden en NSAIDs is algemeen lager bij vogels dan bij zoogdieren (Tabel 1). Maar er zijn enkele uitzonderingen. Salicylzuur heeft bij de meeste vogelspecies een hoger distributievolume dan bij zoogdieren (Hardie et al., 1985; Short et al., 1990; Waters et al., 1993; Landoni en Lees, 1995; Welsh et al., 1993; Baert en De Backer, 2003). Het distributievolume voor carboplatine is bij de verschillende vogelsoorten van dezelfde grootteorde, maar kan grondig verschillen voor zowel NSAIDs als aminoglycosiden (Antonissen et al., 2015) (Tabel 1). Flunixine en meloxicam hebben bij de struisvogel (Struthio camelus) een hoger distributievolume dan bij de kalkoen (Meleagris gallopavo), terwijl dit bij salicylzuur omgekeerd is (Baert en De Backer, 2003).

De lichaamsklaring van aminoglycosiden gebeurt bij vogels sneller dan bij zoogdieren, met uitzondering van gentamicine dat trager wordt geklaard. De lichaamsklaring van NSAIDs varieert echter sterk (Tabel 1). Salicylzuur en meloxicam worden bij vogels aan gelijkaardige snelheid geklaard als bij zoogdieren, uitgezonderd bij de kalkoen die salicylzuur sneller klaart en bij de struisvogel en de kip (Gallus gallus) die meloxicam sneller, respectievelijk trager, klaren (Short et al., 1990; Lees et al., 1991; Waters et al., 1993; Baert en De Backer, 2003). Flunixine wordt daarentegen sneller geklaard bij vogels dan bij zoogdieren, uitgezonderd bij de duif (Columba livia) en de kip. De klaring van flunixine bij de duif gebeurt even snel als bij zoogdieren, terwijl flunixine bij de kip trager geklaard wordt dan bij zoogdieren (Hardie et al., 1985; Landoni en Lees, 1995; Welsh et al., 1993; Baert en De Backer, 2003). De vergelijking tussen verschillende vogelsoorten toont aan dat flunixine en meloxicam bij de struisvogel sneller worden geklaard dan bij de kip, terwijl dit bij salicylzuur tegengesteld is (Baert en De Backer, 2003). De klaring van carboplatine gebeurt aan gelijkaardige snelheid bij de verschillende vogelsoorten, maar verschilt voor zowel NSAIDs als aminoglycosiden (Antonissen et al., 2015; Baert en De Backer, 2003; Dinev, 2008) (Tabel 1).

De eliminatiehalfwaardetijd van aminoglycosiden en NSAIDs varieert sterk tussen zoogdieren en vogels, en tussen vogelsoorten onderling ten gevolge van verschillen in distributievolume en klaring (Tabel 1). Van flunixine, meloxicam, amikacine en kanamycine is de eliminatiehalfwaardetijd bij vogels korter dan bij zoogdieren, met uitzondering van flunixine, meloxicam en kanamycine bij de kip (Hardie et al., 1985; Lees et al., 1991; El-Gammal et al., 1992; Lashev et al., 1992; Welsh et al., 1993; Landoni en Lees, 1995; Baert en De Backer, 2003; Dinev, 2008). Salicylzuur heeft bij vogels en zoogdieren een gelijkaardige eliminatiehalfwaardetijd, uitgezonderd bij de duif en de eend (Anas platyrhynchos) die salicylzuur trager elimineren (Short et al., 1990; Waters et al., 1993; Baert en De Backer, 2003). Gentamicine heeft bij vogels een langere eliminatiehalfwaardetijd dan bij zoogdieren (Pedersoli et al., 1990; Dinev, 2008) en apramycine heeft een sterk variërende eliminatiehalfwaardetijd bij zowel de verschillende vogelspecies als bij zoogdierspecies (Lashev et al., 1992; Haritova et al., 2004; Dinev, 2008). Tussen vogelsoorten zijn de grootste verschillen in eliminatiehalfwaardetijd aanwezig voor NSAIDs, waarbij de duif en de kip een duidelijk langere en de struisvogel een duidelijk kortere eliminatiehalfwaardetijd hebben (Baert en De Backer, 2003) (Tabel 1). Voor aminoglycosiden en carboplatine zijn de verschillen in eliminatiehalfwaardetijd tussen vogelsoorten duidelijk beperkter (Antonissen et al., 2015; Dinev, 2008).

\section{BIOTRANSFORMATIE VAN GENEESMIDDELEN BIJ VOGELS EN VERSCHILLEN MET ZOOG- DIEREN}

De belangrijkste fase I-biotransformatiereactie is de microsomale oxidatie die voornamelijk plaatsvindt in hepatocyten en hoofdzakelijk gemedieerd is door het cytochroom P450 enzymcomplex (Baggot, 1995). De concentraties van cytochroom P450-enzymen in 
Tabel 1. Farmacokinetische parameters van enkele niet-sterö̈dale, anti-inflammatoire geneesmiddelen (NSAIDs), antibiotica en carboplatine na intraveneuze toediening bij zoogdieren en vogels.

\begin{tabular}{|c|c|c|c|c|c|}
\hline \multicolumn{6}{|c|}{ NSAIDs } \\
\hline & Diersoort & $\operatorname{Vd}_{\text {area }}(\mathbf{l} / \mathbf{k g})$ & Cl (l/h/hg) & $\mathbf{T}_{1 / 2 \mathrm{el}}(\mathrm{h})$ & Referentie \\
\hline \multirow[t]{7}{*}{ Salicylzuur } & Hond & 0,285 & 0,041 & 4,49 & Waters, 1993 \\
\hline & Geit & 0,138 & 0,193 & 0,50 & Short, 1990 \\
\hline & Duif & 1,48 & 0,069 & 14,93 & Baert en De Backer, 2003 \\
\hline & Eend & 1,58 & 0,16 & 5,41 & Baert en De Backer, 2003 \\
\hline & Kalkoen & 2,05 & 0,46 & 2,99 & Baert en De Backer, 2003 \\
\hline & Struisvogel & 0,36 & 0,19 & 1,32 & Baert en De Backer, 2003 \\
\hline & Kip & 0,95 & 0,21 & 3,13 & Baert en De Backer, 2003 \\
\hline \multirow[t]{8}{*}{ Flunixine } & Hond & 0,348 & 0,064 & 3,75 & Hardie, 1985 \\
\hline & Paard & 0,317 & 0,058 & 3,37 & Landoni, 1995 \\
\hline & Schaap & 0,166 & 0,060 & 3,82 & Welsh, 1993 \\
\hline & Duif & 0,058 & 0,064 & 0,62 & Baert en De Backer, 2003 \\
\hline & Eend & 0,088 & 0,140 & 0,43 & Baert en De Backer, 2003 \\
\hline & Kalkoen & 0,100 & 0,181 & 0,54 & Baert en De Backer, 2003 \\
\hline & Struisvogel & 0,130 & 0,500 & 0,17 & Baert en De Backer, 2003 \\
\hline & Kip & 0,043 & 0,0009 & 5,52 & Baert en De Backer, 2003 \\
\hline \multirow[t]{6}{*}{ Meloxicam } & Paard & 0,160 & 0,042 & 2,70 & Lees, 1991 \\
\hline & Duif & 0,140 & 0,039 & 2,40 & Baert en De Backer, 2003 \\
\hline & Eend & 0,065 & 0,061 & 0,72 & Baert en De Backer, 2003 \\
\hline & Kalkoen & 0,079 & 0,055 & 0,99 & Baert en De Backer, 2003 \\
\hline & Struisvogel & 0,580 & 0,720 & 0,50 & Baert en De Backer, 2003 \\
\hline & Kip & 0,058 & 0,013 & 3,21 & Baert en De Backer, 2003 \\
\hline \multicolumn{6}{|c|}{ Antibiotica } \\
\hline & Diersoort & Vd $d_{\text {area }}(\mathbf{l} / \mathbf{k g})$ & Cl (l/h/kg) & $\mathbf{T}_{1 / 2 \mathrm{el}}(\mathrm{h})$ & Referentie \\
\hline \multirow[t]{4}{*}{ Gentamicine } & Paard & 86,96 & 0,076 & 2,09 & Dinev, 2008 \\
\hline & Schaap & 11,80 & 0,070 & 1,80 & Dinev, 2008 \\
\hline & Kalkoen & 1,52 & 0,047 & 2,35 & Dinev, 2008 \\
\hline & Kip & 1,08 & 0,047 & 3,38 & Pedersoli et al., 1990 \\
\hline \multirow[t]{4}{*}{ Amikacine } & Paard & 84,53 & 0,074 & 1,71 & Dinev, 2008 \\
\hline & Schaap & 10,31 & 0,062 & 1,79 & Dinev, 2008 \\
\hline & Grijze roodstaartpapegaai & 0,149 & 0,187 & 1,10 & Dinev, 2008 \\
\hline & Kip & 0,763 & 0,109 & 1,44 & El-Gammal et al., 1992 \\
\hline \multirow[t]{4}{*}{ Kanamycine } & Paard & 70,83 & 0,088 & 1,60 & Dinev, 2008 \\
\hline & Schaap & 11,18 & 0,098 & 2,60 & Dinev, 2008 \\
\hline & Duif & 0,088 & 0,214 & 0,92 & Lashev et al., 1992 \\
\hline & Kip & 0,417 & 0,108 & 2,01 & Dinev, 2008 \\
\hline \multirow[t]{4}{*}{ Apramycine } & Schaap & 5,041 & 0,080 & 1,46 & Dinev, 2008 \\
\hline & Duif & 0,023 & 0,210 & 0,25 & Lashev et al., 1992 \\
\hline & Kalkoen & 3,01 & 0,103 & 2,62 & Haritova et al., 2004 \\
\hline & Kip & 0,33 & 0,110 & 1,54 & Dinev, 2008 \\
\hline \multicolumn{6}{|c|}{ Chemotherapeutica } \\
\hline & Diersoort & $V_{d_{\text {area }}}(\mathbf{l} / \mathbf{k g})$ & Cl (l/h/kg) & $\mathbf{T}_{1 / 2 \mathrm{el}}(\mathrm{h})$ & Referentie \\
\hline \multirow[t]{4}{*}{ Carboplatine } & Kip & 0,495 & 0,293 & 1,16 & Antonissen et al., 2015 \\
\hline & Eend & 0,398 & 0,310 & 0,90 & Antonissen et al., 2015 \\
\hline & Duif & 0,517 & 0,521 & 0,68 & Antonissen et al., 2015 \\
\hline & Parkiet & 0,213 & 0,355 & 0,41 & Antonissen et al., 2015 \\
\hline
\end{tabular}


hepatische microsomen zijn lager bij vogels, reptielen en amfibieën dan bij zoogdieren. Bij vogels is deze concentratie het laagst bij visetende vogels en roofvogels (Walker en Ronis, 1989).

Aminopyrine-N-demethylase en anilinehydroxylase zijn beide microsomale fase I-enzymen die verschillen in activiteit tussen vogels en zoogdieren en tussen vogelsoorten onderling. Van beide enzymen is de activiteit bijvoorbeeld lager bij eenden dan bij andere vogelsoorten (kalkoenen, kippen en ganzen) en ratten. De activiteit van aminopyrine-N-demethylase is lager bij eenden dan bij kalkoenen, kippen en ganzen (Anser anser) (Bartlet en Kirinya, 1976).

Via fase II-reacties worden geneesmiddelen of hun fase I-metabolieten met onder andere een hydroxyl-, carboxyl-, amino- of sulfhydrylgroep geconjugeerd met glucuronzuur, acetaat, sulfaat, methyl, gluthatione of bepaalde aminozuren (onder andere ornithine, glycine, glutamine, taurine). De gevormde verbindingen zijn wateroplosbaar en polair en kunnen worden uitgescheiden in de urine of de gal (Williams, 1971). Conjugatiereacties vinden plaats in aanwezigheid van het conjugerend agens, een nucleotide en een transferenzym. Speciesverschillen in conjugatiereacties zijn het gevolg van verschillen in aanwezigheid van elk van deze substanties. Hoewel de fase I-reacties voorkomen bij alle diersoorten, kunnen bepaalde conjugatiereacties deficiënt of afwezig zijn bij bepaalde diersoorten (Baggot, 1995).

Bij zowel vogels als zoogdieren kunnen carboxylgroepen van geneesmiddelen conjugatiereacties ondergaan. Glutamineconjugatie komt uitsluitend voor bij de mens en andere primaatsoorten tijdens de metabolisatie van arylacetinezuur. Taurineconjugatie, vooral voorkomend bij carnivoren, is beperkt tot arylacetinezuur- en cholinezuurderivaten bij deze diersoorten (Kasuya et al., 1999). Ornithineconjugatie komt enkel voor bij reptielen en bepaalde vogels, zoals kippen, eenden, kalkoenen en ganzen (Schachter et al., 1955; Igarashi et al., 1992). Glycineconjugatie komt voor bij alle zoogdieren, maar bij vogels merkwaardig genoeg enkel bij duiven (Schachter et al., 1955). De deficiëntie in glycineconjugatie bij vogels, uitgezonderd bij de duif, is mogelijk het gevolg van de nood aan glycine voor biosynthese van urinezuur, waardoor het onvoldoende beschikbaar is voor biotransformatie (Baldwin et al., 1960; Bridges et al., 1970). Dit kan verklaren waarom de eliminatiehalfwaardetijd van antimicrobiële middelen, carboplatine en NSAIDs, uitgezonderd salicylzuur, bij duiven lager is dan bij kippen.

Tussen vogelsoorten zijn er eveneens verschillen in het aminozuur aangewend bij conjugatiereacties. Met benzoëzuur en 4-aminobenzoëzuur vormen Galliformen voornamelijk ornithineconjugaten, terwijl ze ornithine- en taurineconjugaten vormen met arylacetinezuur. Anseriformen vormen eveneens ornithineconjugaten met benzoëzuur en 4-aminobenzoëzuur. Tot nog toe werden er bij Anseriformen geen studies uitgevoerd omtrent conjugatie van arylacetinezuur. Columbiformen echter vormen glycineconjugaten met benzoëzuur en glycine en/of taurineconjugaten met arylacetinezuur (Huckle et al., 1982; Baert et al., 2004). Conjugatie van fenylacetinezuur gebeurt bij de duif met glycine en taurine en bij de kip met voornamelijk ornithine en in mindere mate glycine (James et al., 1972).

Naast verschillen in aangewend aminozuur zijn er ook verschillen in concentratie van transferenzymen die betrokken zijn bij de conjugatie. Uridine-difosfaatglucuronyltransferase (UDPGT) en sulfotransferase zijn aanwezig in de lever met een lagere activiteit bij ganzen, eenden (aylesbury x pekin) en kippen, dan bij ratten en kalkoenen. Bij khaki-campbelleenden is de activiteit van deze enzymen hoger (Bartlet en Kirinya, 1976).

\section{ALLOMETRISCHE SCHALING: HOE EEN DOSIS EXTRAPOLEREN NAAR ANDERE VOGELSOORTEN IN DE PRAKTIJK?}

De allometrische vergelijking die wordt gebruikt bij het schalen van farmacokinetische parameters is $\mathrm{y}=\mathrm{a}(\mathrm{w})^{\mathrm{b}}$ met $\mathrm{Y}$ als farmacokinetische parameter, $\mathrm{W}$ als lichaamsgewicht, a als allometrische coëfficiënt en $\mathrm{b}$ als allometrische exponent. Hierbij kan de voorspelde klaring van elk geneesmiddel berekend worden op basis van de klaring van het geneesmiddel bij enkele andere zoogdier- of vogelsoorten. De allometrische coëfficiënt en de allometrische exponent worden beide berekend op basis van de regressie van het logaritme van klaring en het logaritme van lichaamsgewicht (Hunter et al., 2008). De allometrische schaling van de klaring van enrofloxacine, gentamicine, salicylzuur, meloxicam en flunixine, gebaseerd op gegevens van verschillende zoogdieren en vogelsoorten, evenals welke zoogdier- en vogelsoorten hiervoor gebruikt werden, wordt weergegeven in Tabel 2.

In Tabel 2 worden naast de voorspelde klaringswaarden eveneens de geobserveerde klaringswaarden voor deze vogelsoorten weergegeven. Het foutpercentage geeft aan in welke mate de voorspelde klaringswaarden afwijken van de geobserveerde en werd berekend als volgt: (Hunter et al., 2008).

$$
\% \text { fout }=\frac{(\text { geobserveerde }- \text { voorspelde } \text { klaring }) * 100}{\text { geobserveerde klaring }}
$$

De voorspelde klaring vanuit zoogdier- (ZD) en vogelgegevens $(\mathrm{V})$ van salicylzuur $(0,012-0,108 \mathrm{l} / \mathrm{h} /$ $\mathrm{kg}$ en $0,148-0,186 \mathrm{l} / \mathrm{h} / \mathrm{kg}$, respectievelijk) en gentamicine $(0,086-0,254 \mathrm{l} / \mathrm{h} / \mathrm{kg}$ en $0,068-0,078 \mathrm{l} / \mathrm{h} / \mathrm{kg}$, respectievelijk) heeft bij extrapolatie tussen vogelsoorten een lager foutpercentage (8-60\% en $18-45 \%$, respectievelijk) dan bij extrapolatie vanuit zoogdieren (43-86\% en $77-319 \%$, respectievelijk). Voor enrofloxacine heeft de voorspelde klaring (0,785-6,182 $1 / \mathrm{h} / \mathrm{kg}$ ZD en $1,506-4,818 \mathrm{l} / \mathrm{h} / \mathrm{kg} \mathrm{V}$ ) een hoog foutper- 
Tabel 2. Geobserveerde versus voorspelde klaringswaarden van enkele geneesmiddelen bij vogels geëxtrapoleerd vanuit zoogdieren en andere vogelsoorten, en de zoogdier- en vogelsoorten die gebruikt werden voor de berekening van de voorspelde klaring per geneesmiddel (Hunter et al., 2008).

\begin{tabular}{|c|c|c|c|c|c|}
\hline \multicolumn{6}{|c|}{ Klaringswaarden van geneesmiddelen bij vogels geëxtrapoleerd vanuit andere zoogdieren } \\
\hline \multicolumn{6}{|c|}{ Antimicrobiële middelen } \\
\hline Geneesmiddel & Diersoort & $\begin{array}{l}\text { Geobserveerse } \\
\text { Cl }(1 / \mathbf{h} / \mathbf{k g})\end{array}$ & $\begin{array}{l}\text { Voorspelde } \\
\mathrm{Cl}(\mathbf{l} / \mathbf{h} / \mathbf{k g})\end{array}$ & $\%$ fout & $\begin{array}{l}\text { Diersoorten gebruikt } \\
\text { voor extrapolatie }\end{array}$ \\
\hline \multirow[t]{3}{*}{ Enrofloxacine } & Kalkoen & 0,435 & 1,224 & 181 & Muis, rat, konijn \\
\hline & Struisvogel & 4,456 & 0,785 & 82 & \\
\hline & Vleeskip & 1,636 & 6,182 & 278 & \\
\hline \multirow[t]{3}{*}{ Gentamicine } & Haan & 0,020 & 0,086 & 319 & Rat, konijn, hond \\
\hline & Kalkoen & 0,027 & 0,076 & 178 & \\
\hline & Roodstaartbuizerd & 0,143 & 0,254 & 77 & \\
\hline \multicolumn{6}{|c|}{ NSAIDs } \\
\hline Geneesmiddel & Diersoort & $\begin{array}{l}\text { Geobserveerse } \\
\text { Cl }(1 / \mathbf{h} / \mathbf{k g})\end{array}$ & $\begin{array}{l}\text { Voorspelde } \\
\text { Cl (l/h/kg) }\end{array}$ & $\%$ fout & $\begin{array}{l}\text { Diersoorten gebruikt } \\
\text { voor extrapolatie }\end{array}$ \\
\hline \multirow[t]{5}{*}{ Salicylzuur } & Duif & 0,069 & 0,012 & 83 & Konijn, hond, geit \\
\hline & Eend & 0,160 & 0,038 & 76 & \\
\hline & Kalkoen & 0,460 & 0,066 & 86 & \\
\hline & Struisvogel & 0,190 & 0,108 & 43 & \\
\hline & Kip & 0,210 & 0,031 & 85 & \\
\hline \multirow[t]{5}{*}{ Meloxicam } & Duif & 0,039 & 0,047 & 21 & Muis, rat, cavia \\
\hline & Eend & 0,061 & 0,057 & 7 & \\
\hline & Kalkoen & 0,055 & 0,063 & 15 & \\
\hline & Struisvogel & 0,720 & 0,069 & 90 & \\
\hline & Kip & 0,013 & 0,055 & 317 & \\
\hline \multirow[t]{5}{*}{ Flunixine } & Duif & 0,064 & 0,296 & 363 & Konijn, kat, hond \\
\hline & Eend & 0,140 & 0,114 & 19 & \\
\hline & Kalkoen & 0,180 & 0,070 & 61 & \\
\hline & Struisvogel & 0,499 & 0,045 & 91 & \\
\hline & Kip & 0,009 & 0,134 & 1385 & \\
\hline \multicolumn{6}{|c|}{ Klaringswaarden van geneesmiddelen bij vogels geëxtrapoleerd vanuit andere vogelsoorten } \\
\hline \multicolumn{6}{|c|}{ Antimicrobiële middelen } \\
\hline Geneesmiddel & Diersoort & $\begin{array}{l}\text { Geobserveerse } \\
\text { Cl }(\mathbf{l} / \mathbf{h} / \mathbf{k g})\end{array}$ & $\begin{array}{l}\text { Voorspelde } \\
\mathrm{Cl}(\mathbf{l} / \mathbf{h} / \mathbf{k g})\end{array}$ & $\%$ fout & $\begin{array}{l}\text { Diersoorten gebruikt } \\
\text { voor extrapolatie }\end{array}$ \\
\hline \multirow[t]{2}{*}{ Enrofloxacine } & Kalkoen & 0,435 & 1,506 & 246 & Havik, nandoe, struisvogel \\
\hline & Vleeskip & 1,636 & 4,818 & 194 & \\
\hline \multirow[t]{2}{*}{ Gentamicine } & Roodstaartbuizerd & 0,143 & 0,078 & 45 & Uil, kip, kalkoen \\
\hline & Gouden adelaar & 0,083 & 0,068 & 18 & \\
\hline \multicolumn{6}{|c|}{ NSAIDs } \\
\hline Geneesmiddel & Diersoort & $\begin{array}{l}\text { Geobserveerse } \\
\text { Cl }(1 / \mathbf{h} / \mathbf{k g})\end{array}$ & $\begin{array}{l}\text { Voorspelde } \\
\mathrm{Cl}(\mathbf{l} / \mathbf{h} / \mathbf{k g})\end{array}$ & $\%$ fout & $\begin{array}{l}\text { Diersoorten gebruikt } \\
\text { voor extrapolatie }\end{array}$ \\
\hline \multirow[t]{2}{*}{ Salicylzuur } & Kalkoen & 0,460 & 0,186 & 60 & Duif, kip, struisvogel \\
\hline & Eend & 0,160 & 0,148 & 8 & \\
\hline \multirow[t]{2}{*}{ Meloxicam } & Kalkoen & 0,055 & 0,187 & 241 & Duif, kip, struisvogel \\
\hline & Eend & 0,060 & 0,080 & 33 & \\
\hline \multirow[t]{2}{*}{ Flunixine } & Kalkoen & 0,180 & 0,131 & 27 & Duif, kip, struisvogel \\
\hline & Eend & 0,140 & 0,070 & 50 & \\
\hline
\end{tabular}


centage zowel bij extrapolatie vanuit zoogdieren als vanuit andere vogelsoorten (82-278\% respectievelijk $194-246 \%)$. Voor de voorspelde klaring van meloxicam $(0,047-0,069$ 1/h/kg ZD en $0,080-0,1871 / \mathrm{h} / \mathrm{kg} \mathrm{V})$ en flunixine $(0,045-0,296 \mathrm{l} / \mathrm{h} / \mathrm{kg} \mathrm{ZD}$ en $0,070-0,131$ $1 / \mathrm{h} / \mathrm{kg} \mathrm{V}$ ). Daarentegen zijn er grote variaties in foutpercentage, zowel bij extrapolatie vanuit zoogdieren (7-317\% en $33-241 \%$, respectievelijk) als andere vogelsoorten (19-1385\% en $27-50 \%$, respectievelijk) (Hunter et al., 2008).

Het gebruik van allometrische schaling voor het voorspellen van de klaring van een geneesmiddel bij vogels uitgaande van gegevens van zoogdieren leidt vaak tot grote verschillen met de werkelijke waarde. Deze verschillen zijn het gevolg van belangrijke fysiologische en anatomische verschillen tussen zoogdieren en vogels (Frazier et al., 1995). Daarnaast worden niet alle geneesmiddelen bij vogels via dezelfde weg geëlimineerd als bij zoogdieren, wat wel wordt verondersteld bij allometrische schaling. Bij extrapolatie uitgaande van gegevens van andere vogelsoorten zijn deze verschillen beperkter, maar kunnen afhankelijk van het geneesmiddel toch beduidend zijn (Mahmood et al., 2006). Naast klaring kan ook de eliminatiehalfwaardetijd geschat worden door middel van allometrische schaling. Alhoewel dit een hybride farmacokinetische parameter is, bepaald door klaring en distributievolume, zou volgens sommigen de eliminatiehalfwaardetijd de meest geschikte farmacokinetische parameter voor allometrische schaling zijn (Riviere et al., 1997). Dit wordt ondersteund door een studie van Antonissen et al. waarin aangetoond wordt dat na carboplatinetoediening bij parkieten, duiven, eenden en kippen, de correlatie tussen lichaamsgewicht en eliminatiehalfwaardetijd $\left(\mathrm{R}^{2}>0,97\right)$ duidelijk hoger is dan deze voor de farmacokinetische parameter klaring $\left(\mathrm{R}^{2}=0,08\right)$ en distributievolume $\left(\mathrm{R}^{2}=0,79\right)$. Echter, indien ook zoogdieren werden ingesloten daalde de determinatiecoëfficiënt $\left(\mathrm{R}^{2}\right)$ voor de eliminatiehalfwaardetijd naar 0,82 (Antonissen et al., 2015). De determinatiecoëfficiënt is een maat voor het lineaire verband tussen het lichaamsgewicht en de eliminatiehalfwaardetijd van het geneesmiddel.

\section{DISCUSSIE}

De verschillen in farmacokinetiek, onder meer in fase I- en fase II-biotransformatie en renale excretie tussen vogels en zoogdieren wijzen erop dat farmacokinetische gegevens van geneesmiddelen bij zoogdieren nagenoeg niet geëxtrapoleerd kunnen worden naar vogels. Dit wordt bevestigd door grote verschillen in geobserveerde en voorspelde klaringswaarden voor enrofloxacine, gentamicine, salicylzuur, meloxicam en flunixine bij vogels geëxtrapoleerd vanuit zoogdieren. Hoewel de verschillen in geobserveerde en voorspelde klaring voor enkele van deze geneesmiddelen bij vogels geëxtrapoleerd vanuit andere vogelsoorten kleiner zijn dan bij extrapolatie vanuit zoog- dieren, kan toch ook een belangrijke variatie worden vastgesteld. Idealiter worden er farmacokinetische en toxiciteitsgegevens gebruikt voor dosisselectie van een geneesmiddel voor de betreffende vogelsoort om veilig gebruik toe te laten. Indien deze gegevens niet beschikbaar zijn, kan bij voorkeur gebruik gemaakt worden van allometrische schaling uitgaande van farmacokinetische gegevens verkregen bij zoogdieren of andere vogelsoorten, afhankelijk van welke diersoort het laagste foutpercentage heeft. Voor geneesmiddelen waarvan de toxiciteit voor vogels laag is, zoals meloxicam bij onder andere de huiskraai (Corvus splendens), de dikbekkraai (Corvus macrorhynchos) en de treurmaina (Acridotheres tristis) (Swarup et al., 2007) en enrofloxacine bij de grijze roodstaartpapegaai (Psittacus erithacus) (Flammer et al., 2008), kan men voor extrapolatie gebruik maken van farmacokinetische gegevens van zowel zoogdieren als -bij voorkeur- andere vogelsoorten. Voor geneesmiddelen met een relatief nauwe therapeutisch-toxische index bij vogels, zoals flunixine bij onder andere de Afrikaanse lepelaar (Platalea alba), Afrikaanse maraboe (Leptoptilos crumenifer) en kuifseriema (Cariama cristata) (Cuthbert et al., 2007) en gentamicine bij de roodstaartbuizerd (Buteo jamaicensis) (Bird et al., 1983), is het noodzakelijk om gebruik te maken van de farmacokinetische gegevens van de betreffende vogelsoort. Voor salicylzuur is de toxiciteit bij de raaf (Corvus corax), de ooievaar (Ciconia ciconia) en de monniksgier (Aegypius monachus) laag (Cuthbert et al., 2007). Door het gebruik van een beperkt aantal dieren in dit experiment is de betrouwbaarheid van deze gegevens voor salicylzuur echter te beperkt om het te beschouwen als een geneesmiddel met een lage toxiciteit bij vogels. Bijgevolg wordt aangeraden om voor dosisextrapolatie gebruik te maken van farmacokinetische gegevens van een vogelsoort van waaruit extrapolatie naar de betreffende vogelsoort het laagste foutpercentage geeft. De specifieke dosis van elk geneesmiddel voor elke vogelsoort kan niet worden berekend op basis van de beschikbare farmaco-kinetische gegevens, maar de gevolgen voor de doseringsfrequentie kunnen wel ingeschat worden. Voor geneesmiddelen die bij bepaalde vogelsoorten trager worden geklaard dan bij zoogdieren en andere vogelsoorten wordt aangeraden om de doseringsfrequentie voor vogels te verlagen. Dit is het geval bij gentamicine, meloxicam (kip), flunixine (kip) vanuit zoogdiergegevens en flunixine (kip) en meloxicam (kip) vanuit struisvogelgegevens. Hetzelfde geldt voor geneesmiddelen die sterke neveneffecten kunnen hebben, zoals flunixine en gentamicine. Voor geneesmiddelen die bij vogels een gelijkaardige klaring hebben als bij zoogdieren en andere vogelsoorten kan een gelijkaardige doseringsfrequentie worden gebruikt. Dit is het geval voor salicylzuur en meloxicam, uitgezonderd bij de kalkoen, kip, struisvogel en flunixine bij de duif uitgaande van zoogdiergegevens, en carboplatine uitgaande van vogelgegevens. Voor geneesmiddelen die 
bij vogels sneller worden geklaard dan bij zoogdieren kan de doseringsfrequentie verhoogd worden. Dit is het geval voor amikacine, kanamycine, apramicine, salicylzuur (bij de kalkoen), en meloxicam (bij de struisvogel) uitgaande van zoogdiergegevens, en meloxicam (bij de struisvogel) uitgaande van gegevens van kippen. Hoewel flunixine sneller wordt geklaard bij vogels, uitgezonderd bij de duif en de kip, dan bij zoogdieren en sneller bij de struisvogel dan bij de kip, wordt hierbij geen hogere doseringsfrequentie aangeraden wegens de nauwe therapeutisch-toxische index bij vogels.

\section{CONCLUSIE}

Er kan geconcludeerd worden dat er nog steeds een grote behoefte is aan farmacokinetisch onderzoek naar geneesmiddelen bij de verschillende vogelsoorten. In afwezigheid van deze gegevens dient dosisextrapolatie bij voorkeur te gebeuren uitgaande van farmacokinetische gegevens van andere vogelsoorten. Indien deze gegevens niet beschikbaar zijn en het geneesmiddel een brede therapeutisch-toxische marge heeft, wordt gebruik gemaakt van farmacokinetische gegevens van zoogdieren.

\section{REFERENTIES}

Akester A.R. (1967). Renal portal shunts in the kidney of the domestic fowl. Journal of Anatomy 101, 569-594.

Antonissen G., Devreese M., De Baere S., Hellebuyck T., Van de Maele I., Rouffaer L., Stemkens H.J.J., De Backer P., Martel A., Croubels S. (2015). Comparative pharmacokinetics and allometric scaling of carboplatin in different avian species. PLOS ONE 10, 1-11.

Baert K. (2003). Pharmacokinetics and pharmacodynamics of non-steroidal anti-inflammatory drugs in birds. Doctoraatsthesis Faculteit Diergeneeskunde, Merelbeke.

Baert K., De Backer P. (2003). Comparative pharmacokinetics of three non-steroidal anti-inflammatory drugs in five bird species. Comparative Biochemistry and Physiology Part C 134, 25-33.

Baert K., Croubels S., Maes A., Hillaert U., Van Calenbergh S., De Backer P. (2004). Comparitive metabolic excretion profile of sodium salicylate in broiler chickens and homing pigeons. Journal of Veterinary Pharmacology and Therapeutics 27, 123-127.

Baggot J.D. (1995). Pharmacokinetics: disposition and fate of drugs in the body. In: Adams R. (editor). Veterinary Pharmacology and Therapeutics. Zevende editie, Iowa State University Press, Ames, p. 18-52.

Baldwin B.C., Robinson D., Williams R.T. (1960). Studies in detoxication. 82. The fate of benzoic acid in some domestic and other birds. Biochemical Journal 76, 595-600.

Bartlet A.L., Kirinya L.M. (1976). Activities of mixed function oxidases, UDP-glucuronyl transferase and sulphate conjugation enzymes in Galliformes and Anseriformes. Quarterly Journal of Experimental Physiology 61, 105-119.
Bird J.E., Walser M.M., Duke G.E. (1983). Toxicity of gentamicin in red-tailed hawks. American Journal of Veterinary Research 44, 1289-1293.

Boxenbaum, H., Fertig, J.B. (1984). Scaling of antipyrine intrinsic clearance of unbound drug on 15 mammalian species. European Journal of Drug Metabolism and Pharmacokinetics 9, 177-183.

Braun E.J., Dantzler W.H. (1972). Function of mammalian-type and reptilian-type nephrons in kidney of desert quail. American Journal of Physiology 222, 617-629.

Bridges J.W., French M.R., Smith R.L., Williams R.T. (1970). The fate of benzoic acid in various species. Biochemical Journal 118, 47-51.

Cuthbert R., Parry-Jones J., Green R.E., Pain D.J. (2007). NSAIDs and scavenging birds: potential impacts beyond Asia's critically endangered vultures. Biology Letters 3, 90-93.

Devriese L.A., Dutta G.N. (1984). Effects of erythromycininactivating Lactobacillus crop flora on blood levels of erythromycin given orally to chicks. Journal of Veterinairy Pharmacology and Therapeutics 7, 49-53.

Dinev T.G. (2008). Comparison of the pharmacokinetics of five aminoglycoside and aminocyclitol antibiotics using allometric analysis in mammal and bird species. Research in Veterinary Science 84, 107-118.

Dorrestein G.M., Van Miert A.S.J.P.A.M. (1988). Pharmacotherapeutic aspects of medication of birds. Journal of Veterinairy Pharmacology and Therapeutics 11, 33-44.

Dorrestein G.M. (1991). The pharmacokinetics of avian therapeutics. Veterinary Clinics of North America: Small Animal Practice 21, 1241-1264.

El-Gammal, A.A., Ravis, W.R., Krista, L.M., Tolbert, D.S., Saad, A., (1992). Pharmacokinetics and intramuscular bioavailability of amikacin in chickens following single and multiple dosing. Journal of Veterinary Pharmacology and Therapeutics 15, 133-142.

Flammer K., Aucoin D.P., Whitt D.A. (2008). Intramuscular and oral disposition of enrofloxacin in African grey parrots following single and multiple doses. Journal of Veterinary Pharmacology and Therapeutics 14, 359-366.

Frazier, D.L., Jones, M.P., Orosz, S.E. (1995). Pharmacokinetic considerations of the renal system in birds: part $\mathrm{i}$. anatomic and physiologic principles of allometric scaling. Journal of Avian Medicine and Surgery 9, 92-103.

Gibaldi M., Perrier D. (1975). Pharmacokinetics. In: J. Swarbrick (editor). Drugs and the Pharmaceutical Sciences. Second edition, University of North Carolina, Chapel Hill, p. 1-112.

Goldstein D.L., Skadhauge E. (2000). Renal and extrarenal regulation of body fluid composition. In: G.C. Whittow (editor). Sturkie's Avian Physiology. Fifth edition, Academic press, London, p. 265-298.

Hardie E.M., Hardee G.E., Rawlings C.A. (1985). Pharmacokinetics of flunixin meglumine in dogs. American Journal of Veterinary Research 46, 235-237.

Haritova, A., Lashev, L., (2004). Pharmacokinetics of amikacin in lactating sheep. Veterinary Research Communications 28, 429-435.

Huckle K.R., Stoydin G., Hutson D.H., Millburn P. (1982). Formation of an N-acetylornithine conjugate of 3-phenoxybenzoic acid in the chicken. Drug Metabolism and Disposition 10, 523-528.

Hunter R.P., Mahmood I., Martinez M.N. (2008). Prediction of xenobiotic clearance in avian species using mammalian of avian data: how accurate is the prediction? 
Journal of Veterinary Pharmacology and Therapeutics 31, 281-284.

Igarashi K., Suzuki R., Kasuya F., Fukui M. (1992). Determination of ornithine conjugates of some carboxylic acids in birds by high-performance liquid chromatography. Chemical and Pharmaceutical Bulletin 40, 21962198.

James M.O., Smith R.L., Williams R.T., Reidenberg M. (1972). The Conjugation of phenylacetic acid in man, sub-human primates and some non-primate species. Proceedings of the Royal Society of London. Series B, Biological Sciences 182, 25-35.

Jerzsele A. (2012). Comparative veterinary pharmacokinetics. In: Noreddin A. (editor). Readings in Advanced Pharmacokinetics - Theory, Methods and Applications. Intech, Rijeka, p. 179-198.

Kasuya F., Igarashi K., Fukui M. (1999). Characterization of a renal medium chain acyl-CoA synthetase responsible for glycine conjugation in mouse kidney mitochondria. Chemico-Biological Interactions 118, 233-246.

Landoni M.F., Lees P. (1995). Comparison of the anti-inflammatory actions of flunixin and ketoprofen in horses applying PK/PD modelling. Equine Veterinary Journal 27, 247-256.

Lashev, L.D., Pashov, D.A., Marinkov, T.N., (1992). Interspecies differences in the pharmacokinetics of kanamycin and apramycin. Veterinary Research Communications 16, 293-300.

Lees P., AliAbadi F.S. (2002). Rational dosing of antimicrobial drugs: Animals versus humans. International Journal of Antimicrobial Agents 19, 269-284.

Lees P., Sedgewick A.D., Higgins A.J., Pugh K.E., Busch U. (1991). Pharmacodynamics and pharmacokinetics of miloxicam in the horse. British Veterinary Journal 147, 97-108.

Mahmood, I., Martinez, M., Hunter, R.P. (2006). Interspecies allometric scaling. Part I: prediction of clearance in large animals. Journal of Veterinary Pharmacology and Therapeutics 29, 415-423.

Obach, R.S., Baxter, J.G., Liston, T.E., Silber, B.M., Jones, B.C., Macintyre, F. (1997). The prediction of human pharmacokinetic parameters from preclinical and in vitro metabolism. Journal of Pharmacology and Experimental Therapeutics, 283, 46-58.

Pedersoli, W.M., Ravis, W.R., Askins, D.R., Krista, L.M., Spano, J.S., Whitesides, J.F. (1990). Pharmacokinetics of single-dose intravenous or intramuscular administration of gentamicin in roosters. American Journal of Veterinary Research, 51, 286-289.

Riviere, J.E. (2011). Comparative Pharmacokinetics: Principles, Techniques and Applications. Second edition, John Wiley and Sons, Iowa State University Press.
Riviere J.E., MartinJiminez T., Sundlof S.F., Craigmill A.L. (1997). Interspecies allometric analysis of the comparative pharmacokinetics of 44 drugs across veterinary and laboratory animal species. Journal of Veterinary Pharmacology and Therapeutics 20, 453-463.

Rowland M., Tozer T.N. (1995). Absorption and disposition. In: Clinical Pharmacokinetics: Concepts and Applications. Third edition, Lippincott Williams and Wilkins, Pensylvania, p. 11-52.

Schachter, D., Manis, J. G., Taggart, J. V. (1955). Renal synthesis, degradation and active transport of aliphatic acyl amino acids. American Journal of Physiology-Legacy Content 182, 537-544.

Shargel L., Wu-Pong S., Yu A.B.C. (2004). Drug elimination and clearance en pharmacokinetics of oral absorption. In: Applied Biopharmaceutics and Pharmacokinetics. Fifth edition, McGraw-Hill Education, New York, p. 96-136.

Short C.R., Hsieh L.C., Malbrough M.S., Barker S.A., Neff-Davis C.A., Davis L.E. (1990). Elimination of salicylic acid in goats and cattle. American Journal of Veterinary Research 8, 1267-1270.

Swarup D., Patra R.C., Prakash V., Cuthbert R., Das D., Avari P. et al (2007). Animal Conservation 10, 192-198.

Toutain P.L., Ferran A., Bousquet-Mélou A. (2010). Species differences in pharmacokinetics and pharmacodynamics. In: Cunningham F., Elliot J., Lees P. (editors). Comparative and Veterinary Pharmacology, Handbook of Experimental Pharmacology. Volume 199, SpringerVerslag, Berlin Heidelberg, p. 19-48.

Vermeulen B., De Backer P., Remon J.P. (2002). Drug administration to poultry. Advanced Drug Delivery Reviews 54, 795-803.

Walker C.H., Ronis M.J. (1989). The monooxygenases of birds, reptiles and amphibians. Xenobiotica, 19, 11111121 .

Waters D.J., Bowers L.D., Cipolle R.J., Caywood D.D., Bills R.L. (1993). Plasma salicylate concentrations in immature dogs following aspirin administration: comparison with adult dogs. Journal of Veterinary Pharmacology and Therapeutics 16, 275-282.

Welsh E.M., Mc Kellar Q.A., Nolan A.M. (1993). The pharmacokinetics of flunixin meglumine in the sheep. Journal of Veterinary Pharmacology and Therapeutics, 16, 181-188.

Williams R.T. (1971). Species variations in drug biotransformations. In: LaDu B.N., Mandel H.G., Way E.L. (editors). Fundamentals of Drug Metabolism and Drug Disposition, Williams \& Wilkins, Baltimore, p. 187. Geciteerd door Baggot J.D. (1995). 